\title{
Comparação de comportamentos maternos e infantis diferenciados pelo sexo, escolaridade e problemas de comportamento
}

\section{Comparison of maternal and child behaviors differentiated by sex, education and behavior problems}

\author{
Jéssica Aline Rovaris (orcid.org/0000-0003-4885-2213) ${ }^{1}$ \\ Alessandra Turini Bolsoni-Silva (orcid.org/0000-0002-0286-780X) ${ }^{2}$
}

\begin{abstract}
Resumo
Este estudo buscou descrever e comparar práticas educativas e comportamentos infantis em crianças diferenciadas por problema de comportamento, escolaridade e sexo. Participaram 155 mães de crianças da rede pública de ensino, em idade pré-escolar ou escolar, de ambos os sexos, que responderam aos instrumentos CBCL, RE-HSE-P. Os dados foram analisados por meio do teste $t$ de Student. O estudo encontrou que meninos e crianças com problemas de comportamento são mais expostas às práticas negativas e as mães se queixam mais frequentemente de seus comportamentos. Por outro lado, as meninas obtiveram maiores médias para habilidades sociais e práticas positivas. Foram encontradas práticas específicas e comportamentos infantis mais frequentes para grupos diferenciados pelo sexo ou pelo diagnóstico de problemas de comportamento, tanto do ensino infantil quanto do fundamental. Análises como essas podem colaborar para o desenvolvimento de estratégias de intervenção para grupos focais.
\end{abstract}

Palavras-chave: Comportamento materno. Práticas educativas. Habilidades sociais. Problema de comportamento. Relações entre comportamentos.

\begin{abstract}
:
\footnotetext{
${ }^{1}$ Universidade Estadual Paulista, São Paulo, Brasil. E-mail: jessica.rovaris@gmail.com.

${ }^{2}$ Universidade Estadual Paulista, São Paulo, Brasil. E-mail: bolsonisilva@gmail.com.
}

This study aimed to describe and compare educational practices and child behaviors in children differentiated by behavior problem, education and sex. The participants were 155 mothers of children from the public school system, in preschool and school age, of both sexes, who answered the CBCL, RE-HSE-P instruments. The data were analyzed through test $\mathrm{t}$ of Student. The study found that boys and children with behavior problems are more exposed to negative practices and mothers complain more often about their behaviors. In contrast, the girls obtained higher averages for social skills and positive practices. Specific practices and more frequent child behaviors 
were found for groups differentiated by sex or diagnosis of behavioral problems, both in early childhood education and elementary school. Such analyzes can contribute to the development of intervention strategies for focus groups.

Keywords: Maternal behavior. Educational practices. Social skills. Behavior problem. Relationships between behaviors.

A literatura da área do desenvolvimento (Bartholomeu, Montiel, Flamenghi \& Machado, 2016; Bolsoni-Silva, Loureiro \& Marturano, 2016; Kårstad, Kvello, Wichstrøm \& Berg-Nielsen, 2013; Sierra \& Veja, 2014) é consistente ao afirmar que as práticas educativas parentais interferem diretamente nos comportamentos desenvolvidos pelas crianças no seu curso de vida. Os pais podem adotar práticas que favoreçam o desenvolvimento saudável dos filhos e os auxilie a aprender habilidades sociais (Danket et al., 2016; Kårstad et al., 2013; Leme \& Marturano, 2014;Torre, Casanova, Carpio \& Cerezo, 2013) ou, ao contrário, podem assumir práticas que dificultem a relação familiar e aumente a probabilidade de que as crianças desenvolvam problemas de comportamento (Bolsoni-Silva et al., 2016; Sierra \& Veja, 2014; Teixeira, Marino \& Carreiro, 2015).

Conceitualmente, práticas educativas parentais são as estratégias utilizadas pelos pais com fins a orientar os filhos para a aquisição de alguns comportamentos e supressão de outros, considerados como socialmente desfavoráveis ou inadequados (Newcobe, 1999). Segundo Gomide (2006), as práticas educativas são consideradas positivas quando há o uso contingente da atenção, da distribuição de privilégios, do estabelecimento de regras, além do contínuo oferecimento de afeto, do acompanhamento e da supervisão das atividades dos filhos e das ações de ordem moral. Mas, quando há comportamentos negligentes por parte dos pais, falta de atenção e de afeto, abuso físico e psicológico, uso de ameaças, chantagens, humilhação e a monitoria negativa, trata-se das práticas negativas.

No estudo de Torre et al. (2013), adolescentes agressivos, que apresentavam pouca satisfação e bem-estar, tinham também pais pouco afetuosos, pouco comunicativos, impositivos, severos a respeito de regras e que utilizavam pouca monitoria positiva. Os pesquisadores encontraram, portanto, relação entre as práticas negativas dos pais e os 
problemas de comportamento dos adolescentes, indo ao encontro dos dados de Gomide (2006).

A respeito das práticas positivas, Alvarenga, Weber e Bolsoni-Silva (2016) e BolsoniSilva et al. (2016) afirmam que o repertório de habilidades sociais dos pais pode ser determinante na adoção de tais práticas. Bolsoni-Silva (2003) constatou a existência de algumas classes de comportamentos habilidosos mais relevantes na relação com os filhos e que, portanto, interferem de forma consistente na adoção das práticas positivas; a esse repertório, ela denominou Habilidades Sociais Educativas Parentais (HSE-P).

No estudo de Kim, Doh, Hong e Choi (2011), o aumento das habilidades sociais impactou na diminuição das práticas negativas e problemas de comportamento. Para isso, os pesquisadores conduziram um treinamento em habilidades sociais com crianças que apresentavam comportamentos agressivos e suas mães. Os resultados demonstraram que, após o treino, houve a diminuição das práticas negativas maternas e dos modelos comportamentais agressivos para as crianças. Desse modo, à medida que as habilidades sociais das mães melhoravam, melhorava também sua relação com a criança; além disso, houve o aumento do repertório de habilidades sociais das crianças e a diminuição dos seus comportamentos agressivos (Kim et al., 2011).

Os estudos de Kim et al. (2011), de Alvarenga et al. (2016) e de Bolsoni-Silva et al. (2016) atestam que pais mais habilidosos conseguem ensinar mais habilidades sociais para os filhos. Constatar essa relação é relevante, pois, segundo Patias, Siqueira e Dias (2013), as habilidades sociais infantis são consideradas fator de proteção ao desenvolvimento de problemas de comportamento, por auxiliar a criança a lidar com demandas próprias da interação social e favorecer a resolução de problemas, por exemplo.

$\mathrm{Na}$ direção oposta, estão os problemas de comportamento, que, além de serem obstáculos ao desenvolvimento saudável da criança, também são preditores de outros problemas graves na adolescência ou idade adulta, como transtornos comportamentais, comportamento delinquente e dificuldades acadêmicas (Teixeira et al., 2015; Torre et al., 2013). Eles podem ser definidos como deficit ou excessos comportamentais, que se 
manifestam na relação da criança com pares e adultos e dificultam o acesso delas às contingências de reforçamento (Bolsoni-Silva, 2003).

Os problemas de comportamento podem ser de dois tipos: externalizantes e internalizantes. O primeiro refere-se à agressividade física ou verbal, comportamentos antissociais, hiperatividade, desobediência frequente, roubo, ameaça, acessos de raiva e impulsividade (Achenbach \& Rescorla, 2001). Já os internalizantes podem ser exemplificados pela depressão, isolamento social, ansiedade (Achenbach \& Rescorla, 2001). Devido às dificuldades que os problemas de comportamento causam às crianças, estudos (Bolsoni-Silva, Marturano \& Freira, 2010; Bolsoni-Silva \& Loureiro, 2016; Pizato, Marturano \& Fontaine, 2014) têm buscado encontrar outras variáveis que podem interferir no seu desenvolvimento.

O estudo de Pizato et al. (2014) constatou uma trajetória positiva para habilidades sociais durante o ciclo escolar para meninos e meninas, diferentemente de Bolsoni-Silva et al. (2010), cujo estudo declara que o aumento das habilidades sociais ocorreu apenas para grupos específicos (meninas, crianças com problemas de comportamento). Por outro lado, Reynolds, Sander e Irvin (2010) encontraram relação inversa: aumento dos problemas de comportamento nas séries escolares mais avançadas, e Konold, Jamison e Stanter-Chapman (2010) mais habilidades sociais para crianças menores. Apesar de a possibilidade de diferenças metodológicas terem atuado sob os resultados das pesquisas, pode-se afirmar que a literatura não é consistente quanto aos achados relacionados à interferência da idade escolar nos comportamentos infantis, o que se constitui uma lacuna na área.

Nesse campo, Bolsoni-Silva e Loureiro (2016) buscaram verificar se há habilidades sociais específicas mais comuns às crianças do ensino infantil e fundamental. As autoras encontraram que crianças do ensino infantil costumavam negociar, comunicar-se, expressar carinho e direitos mais frequentemente; já as crianças em idade escolar conseguiam expressar frustração, desejos, preferências e tomar iniciativa. Assim, apesar da falta de concordância a respeito do repertório comportamental de crianças de diferentes idades, a pesquisa de Bolsoni-Silva e Loureiro (2016) demonstrou que ambos os grupos têm habilidades sociais e que estas podem diferenciá-los. 
Nessa direção, os estudos afirmam que os meninos costumam apresentar mais problemas de comportamento, se comparados às meninas, compondo, portanto, mais frequemente os grupos clínicos (Bolsoni-Silva et al., 2016; Comôdo, Del Prette \& Del Prette, 2017). Ao contrário, as meninas são identificadas como mais habilidosas e com menos problemas de comportamento. Essas relações indicam que, provavelmente, as meninas são expostas a fatores de proteção para os problemas de comportamento mais intensamente que os meninos e, portanto, aprendem mais habilidades sociais e tornam-se mais sociáveis (Leman \& Bjornberg, 2010; Marturano, Toller \& Elias, 2005).

Os resultados de Marin, Piccinini, Ribeiro e Tudge (2012) apontaram nessa direção. As mães demonstraram mais autocontrole e competência social na relação com as meninas que estavam na primeira infância (ensino infantil), por outro lado, as práticas coercitivas maternas foram relacionadas aos problemas de comportamento, especialmente externalizantes.

Há evidência, portanto, da forte influência das práticas maternas sobre o comportamento das crianças e que pode haver diferenças nas práticas específicas que elas, as mães, utilizam com filhos e filhas (Comôdo et al., 2017; Marin, et al., 2012). No entanto, Bolsoni-Silva (2017) afirma que a diferença nas práticas educativas parece estar mais relacionada aos comportamentos que as crianças emitem do que ao sexo delas, propriamente dito. Desse modo, como os meninos costumam apresentar mais problemas de comportamento, pode ser que as mães tenham mais dificuldade de serem positivas com eles, sendo assim, estudos que busquem investigar práticas específicas com diferentes grupos de crianças podem auxiliar na obtenção de respostas para tais questões.

Finalmente, as pesquisas encontradas apontaram para a importância das habilidades sociais na relação entre mães e crianças, constituindo-se fator de proteção aos problemas de comportamento (Alvarenga et al., 2016; Bolsoni-Silva et al., 2016; Kim et al., 2011 ; Torre et al., 2013). Ademais, variáveis como a fase escolar (Bolsoni-Silva et al., 2010; Konold et al., 2010; Pizato et al., 2014; Reynolds et al., 2010), sexo da criança (Bolsoni-Silva et al., 2015) e emissão de problemas de comportamento (Bolsoni-Silva, 2017) podem interferir no 
repertório infantil, bem como nas práticas educativas maternas, havendo, portanto, reciprocidade entre os comportamentos maternos e infantis (Kim et al., 2011).

À parte das constatações dos estudos citados, alguns dados ainda são inconclusivos. Pizato et al. (2014) afirmaram que as crianças do ensino fundamental tendem a ser mais habilidosas que as do ensino infantil, porém, Bolsoni-Silva et al. (2010) verificaram que essa relação pode estar condicionada a subgrupos específicos (como o das meninas ou das crianças sem problemas de comportamento clínico), mas outros estudos encontraram relação inversa (Konold et al., 2010; Reynold et al., 2010), ou seja, mais habilidades sociais e menos problemas de comportamento para crianças do ensino infantil. Por outro lado, Bolsoni-Silva e Loureiro (2016) verificaram habilidades sociais para crianças dos dois grupos, mas referentes a classes distintas. Levando-se em conta que os comportamentos de mães e crianças são funcionalmente relacionados, Marin et al. (2012) encontraram diferenças entre as práticas educativas de mães aplicadas a meninos e meninas, mas Bolsoni-Silva (2017) encontrou empiricamente que a diferença nas práticas maternas estava condicionada aos comportamentos que as crianças emitiam, e não ao sexo delas. É nesse ínterim que a presente pesquisa se insere.

Este estudo pretende ajudar a preencher tais lacunas respondendo à seguinte questão: há diferenças nas práticas educativas maternas, nas habilidades sociais e queixas de problemas de comportamento de crianças do ensino infantil e fundamental, diferenciadas pelo sexo ou pelo diagnóstico de problemas de comportamento?

Então, o objetivo deste estudo é o de comparar os problemas de comportamento clínico de crianças do ensino infantil (EI) e fundamental (EF) e, em seguida, comparar os comportamentos que compõem as grandes categorias de comportamentos do Roteiro de Habilidades Sociais Educativas Parentais (HSE-P), diferenciando grupos clínicos e não clínicos para problemas de comportamento, meninos e meninas, também do ensino infantil e fundamental. 


\section{Método}

\section{Participantes}

Participaram do estudo 155 mães de crianças matriculadas na rede pública de ensino de uma cidade do Centro-Oeste do estado de São Paulo. Destas, 79 eram mães de crianças do ensino infantil e 76 mães de crianças do ensino fundamental.

Tabela 1. Dados demográficos

\begin{tabular}{|c|c|c|c|c|c|c|}
\hline Idade Média & \multicolumn{2}{|c|}{ Estado Civil } & \multicolumn{2}{|l|}{ Escolaridade } & \multicolumn{2}{|c|}{ Renda familiar* } \\
\hline \multirow[t]{7}{*}{$\begin{array}{l}27,8 \text { anos } \\
(\mathrm{DP}=12,4)\end{array}$} & Casadas & $61,2 \%$ & $\begin{array}{l}\text { Ensino Fundamental } \\
\qquad\left.\right|^{* *}\end{array}$ & $34,8 \%$ & $\begin{array}{l}\text { Até um } \\
\text { salário }\end{array}$ & $27 \%$ \\
\hline & União estável & $16,1 \%$ & $\begin{array}{l}\text { Ensino Fundamental } \\
\qquad \|^{* * * *}\end{array}$ & $46,4 \%$ & Dois salários & $35,4 \%$ \\
\hline & Solteiras & $16,7 \%$ & Ensino Médio & $17,4 \%$ & Três salários & $21,2 \%$ \\
\hline & Divorciadas & $3,22 \%$ & Não informada & $1,7 \%$ & $\begin{array}{l}\text { Quatro } \\
\text { salários }\end{array}$ & $4,5 \%$ \\
\hline & Viúvas & $0,6 \%$ & & & $\begin{array}{l}\text { Cinco } \\
\text { salários }\end{array}$ & $3,2 \%$ \\
\hline & $\begin{array}{c}\text { Não } \\
\text { informado }\end{array}$ & $0,6 \%$ & & & $\begin{array}{l}\text { A partir de } \\
\text { seis salários }\end{array}$ & $5,16 \%$ \\
\hline & & & & & $\begin{array}{l}\text { Não } \\
\text { informada }\end{array}$ & $2,5 \%$ \\
\hline
\end{tabular}

Legenda: *em salários mínimos; ** Até o quinto ano; ***A partir do quinto ano.

Fonte: Elaborada pelas autoras.

Instrumentos

O Child Behavior Checklist - CBCL (Inventários de Comportamentos da Infância e Adolescência, Achenbach \& Rescorla, 2001) para pré-escolares e escolares (1,5 a 5 anos e 6 
a 18 anos), que investigam, a partir do relato de pais, a frequência de 113 ou 118 (a depender da faixa etária) respostas indicativas de problemas de comportamento por meio dos escores "não é verdadeiro $=0$ ", "um pouco verdadeiro $=1$ " e "muito verdadeiro = 2" e de perguntas relacionadas à vida escolar, social e familiar da criança. Os resultados são organizados em problemas internalizantes, externalizantes e totais, que podem ser classificados como clínicos, limítrofes e não clínicos. Os problemas de comportamento são avaliados segundo as seguintes escalas: Escala de Internalização (Ansiedade/Depressão, Retraimento/Depressão, Queixas Somáticas); Escala de Externalização (Violação de Regras e Comportamento Agressivo) e Escala Total de Problemas Emocionais/Comportamentais (Escala de Inter e Externalização em conjunto com Problemas de Sociabilidade, Problemas de Atenção, Problemas com o Pensamento); conforme Achenbach \& Rescorla (2001).

O Roteiro de Entrevista de Habilidades Sociais Educativas Parentais (RE-HSE-P, Bolsoni-Silva et al., 2016) avalia, funcionalmente e em termos de frequência de comportamentos, pais/cuidadores, filhos e variáveis contextuais. Para tal avaliação, o instrumento utiliza perguntas abertas organizadas em grandes categorias que, posteriormente, são subdividas em categorias menores, são elas: 1. Habilidades sociais educativas parentais - HSE-P (relacionada a práticas educativas positivas. 2. Habilidades sociais educativas parentais (HSE-P-conjugal), específicas da interação conjugal. 3. Práticas educativas negativas. 4. Habilidades sociais infantis. 5) Variáveis contextuais contingentes aos comportamentos parentais. O instrumento foi aprovado pelo Conselho Federal de Psicologia e, quanto à consistência interna, apresentou um alpha de 0,846, apresentando dois fatores: total positivo e total negativo e discrimina crianças com e sem problemas de comportamento, escolares de pré-escolares e meninos versus meninas. A partir de Curvas Roc, apresenta pontos de corte para os construtos mensurados no instrumento.

\section{Procedimento de coleta de dados}

Após a aprovação da pesquisa pela Secretaria de Educação e pelo Comitê de Ética em Pesquisa da Universidade Estadual Júlio de Mesquita Filho (CAAE 34642314.4.0000.5398), 
contataram-se as escolas de Educação Infantil (EI) e de Ensino Fundamental (EF) para verificar o interesse em participar do projeto e para, a partir das escolas, ter acesso às famílias das crianças. Nas escolas, os passos foram os seguintes: a) informar os objetivos da pesquisa para a diretora ou a coordenadora pedagógica; b) convidar os professores das crianças a participarem da pesquisa; c) os professores que aceitaram participar indicaram duas crianças, uma que eles julgavam ter problemas de comportamento, outra que julgavam não ter; d) em seguida, as mães dessas crianças foram contatadas por telefone para verificar o interesse em participar da pesquisa.

Com as mães que aceitaram, foi agendado um horário para entrevista, que pôde ser realizada na escola da criança ou na casa das famílias, a critério de escolha das mães. $\mathrm{Na}$ entrevista, as mães assinaram um Termo de Consentimento Livre e Esclarecido e responderam para o filho aos instrumentos $C B C L$ e RE-HSE-P. Como devolutiva, foi perguntado às participantes se elas se interessavam que o filho passasse por atendimento especializado; nas escolas, foram conduzidas palestras informativas sobre práticas educativas e comportamentos infantis e também foram entregues materiais informativos (Bolsoni-Silva, Marturano \& Loureiro, 2011).

\section{Procedimento para tratamento e análise dos dados}

Depois da coleta, os instrumentos foram corrigidos segundo instruções próprias; em seguida, com os dados do CBCL, foi realizada a classificação e categorização das crianças com problemas de comportamento clínico (CPC) e sem problemas de comportamento clínico (SPC); com os resultados do RE-HSE-P, foram realizadas tabulações dos comportamentos, a partir das categorias gerais do instrumento, conforme listado a seguir:

1. Habilidades sociais infantis: conversa, ajuda, oferece atenção, participa, obedece, faz elogios, agradece, assertivo, ${ }^{3}$ brinca, sente-se feliz, desculpa-se e faz carinhos.

2. Práticas positivas: explica, elogia, estabelece regras, conversa/pergunta, agrada, faz

\footnotetext{
3 Assertividade: reunião de comportamentos socialmente esperados em situação de desequilíbrio nas trocas interpessoais, desrespeito ou ameaça de perda de direitos, com a função de restabelecer a condição anterior ou melhorar a condição atual (Del Prette \& Del Prette, 2001).
} 
carinhos, incentiva, brinca, sente-se feliz, tem a mesma opinião do cônjuge, desculpa-se, aceita a opinião do cônjuge e expressa-se longe da criança.

3. Queixa de problemas de comportamento: agride, desobedece, faz birras, responde mal, chora, fica quieto, esquiva-se, ${ }^{4}$ fica tímido, demonstra raiva e desorganizado.

4. Práticas negativas: bate, grita/fica bravo, esquiva-se, sente-se mal, cônjuge é agressivo, casal pensa diferente, não faz nada, expressa-se na presença do filho e briga com a criança.

Em seguida foram conduzidos teste de comparação por meio do teste $t$ de Student para análise estatística, considerando significante $p \leq 0,05$. As crianças com e sem problemas de comportamento foram comparadas diferenciando grupos do El e do EF. Na sequência, as práticas positivas, as habilidades sociais, as práticas negativas e os problemas de comportamentos foram comparados diferenciado-se grupos de meninos e meninas do ensino infantil e fundamental, e posteriormente a grupos com e sem problemas de comportamento ( $\mathrm{CBCL})$, também do ensino infantil e fundamental.

\section{Resultados}

Os resultados foram organizados em nove Tabelas: a Tabela 2 refere-se à comparação das crianças do El e do EF, quanto à presença ou ausência de problemas de comportamento (CBCL). Nas demais Tabelas $(3,4,5,6,7,8,9$ e 10), estão as comparações das habilidades sociais, problemas de comportamento, práticas negativas e práticas positivas para crianças CPC e SPC e para meninos e meninas, constando apenas as categorias específicas para as quais houve significância estatística $(p \leq 0,05)$.

\footnotetext{
4 Esquiva: respostas comportamentais que visam reduzir a incidência de estímulos anteriormente neutros, mas que se tornaram aversivos por meio do condicionamento operante (Dutra, 2010).
} 
Tabela 2. Características das crianças do ensino infantil (EI) e do ensino fundamental (EF) avaliadas pelas mães

\section{${ }^{*}$ Amostra Total $=155$}

\begin{tabular}{|c|c|c|c|c|c|c|c|c|c|c|c|}
\hline \multicolumn{3}{|c|}{ Meninos = $93(60 \%)$} & & \multicolumn{3}{|c|}{ Meninas = 62 (40\%) } & & & \multicolumn{3}{|c|}{$\begin{array}{l}\text { Total (avaliação do } \\
\text { CBCL) }\end{array}$} \\
\hline $\mathrm{CPC} * *$ & 57 & $61 \%$ & & CPC & 22 & $35 \%$ & & & CPC & 79 & $51 \%$ \\
\hline $\mathrm{SPC} * * *$ & 36 & $39 \%$ & & SPC & 40 & $64 \%$ & & & SPC & 76 & $49 \%$ \\
\hline \multicolumn{6}{|c|}{ Ensino Infantil (El) = 79 (51\%) } & \multicolumn{6}{|c|}{ Ensino Fundamental (EF) = 76 (49\%) } \\
\hline & & & $\begin{array}{l}\text { Total por } \\
\text { sexo }\end{array}$ & $\begin{array}{r}\mathrm{Tc} \\
\text { (ava } \\
\text { do } C\end{array}$ & $\begin{array}{l}\text { tal } \\
\text { iação } \\
\text { BCL) }\end{array}$ & & & & $\begin{array}{l}\text { Total por } \\
\text { sexo }\end{array}$ & \multicolumn{2}{|c|}{$\begin{array}{l}\text { (avaliação } \\
\text { do CBCL) }\end{array}$} \\
\hline \multirow{4}{*}{ Meninos } & & 26 & \multirow{4}{*}{$43(54 \%)$} & \multirow{4}{*}{ CPC } & & & & 31 & \multirow{4}{*}{$50(63 \%)$} & \multirow{4}{*}{$\mathrm{CPC}$} & \\
\hline & CPC & $(32 \%)$ & & & 39 & Maningr & CPC & $(41 \%)$ & & & 40 \\
\hline & & 17 & & & $(49 \%)$ & mieninos & & 19 & & & $(52 \%)$ \\
\hline & SPC & $(21 \%)$ & & & & & SPC & $(25 \%)$ & & & \\
\hline \multirow{4}{*}{ Meninas } & & 13 & \multirow{4}{*}{$36(45 \%)$} & \multirow{4}{*}{ SPC } & & & & 9 & \multirow{4}{*}{26 (34\%) } & \multirow{4}{*}{ SPC } & \\
\hline & CPC & $(16 \%)$ & & & 40 & Moninn & CPC & $(12 \%)$ & & & 26 \\
\hline & & 23 & & & $(51 \%)$ & Meninas & & 17 & & & $(34 \%)$ \\
\hline & SPC & (29\%) & & & & & SPC & $(22 \%)$ & & & \\
\hline
\end{tabular}

Legenda: *Quantidade de crianças avaliadas; **Com problemas de comportamento clínico; ***Sem problemas de comportamento clínicos.

Fonte: Elaborada pelas autoras.

A Tabela 2 traz resultados importantes acerca das características das crianças avaliadas. Nas primeiras linhas, há os dados gerais da amostra antes de agrupá-los no El e no EF. Assim, é possível notar que há mais meninos do que meninas no grupo como um todo (cerca de 60\%). Percebe-se também que os meninos são a maioria no grupo com problemas de comportamento clínico (61\%) e as meninas a maioria no grupo SPC (64\%). Contudo, ao se analisar os dados gerais de crianças CPC e SPC, nota-se um valor equiparado: $51 \%$ pertencem a CPC e $49 \%$ pertencem a SPC. 
Na subdivisão da amostra entre El e EF, é possível afirmar também que há uma quantidade semelhante de sujeitos em cada grupo (51\% e $49 \%$, respectivamente). Mesmo após a separação da amostra, os meninos continuam maioria no grupo CPC. Outro dado relevante é que a quantidade de crianças CPC no El e no EF é bastante semelhante ( $n=40$ no El e $\mathrm{n}=39$ no EF), também há alguma diferença no grupo SPC, com maior número para a subamostra do El.

Tabela 3. Habilidades sociais de crianças do ensino infantil (El) e do ensino fundamental (EF), diferenciados pela presença ou ausência de problemas de comportamento

\begin{tabular}{cccccc}
\hline $\begin{array}{c}\text { Categorias específicas do RE-HSE-P } \\
\text { e QRSH-p }\end{array}$ & $\begin{array}{c}\text { CPC** } \\
(\mathbf{n}=79)\end{array}$ & $\begin{array}{c}\text { SPC } \\
(\mathbf{n}=76)\end{array}$ & & \\
\cline { 2 - 6 } & & Média (Desvio Padrão) & $\boldsymbol{t}$ & $\boldsymbol{p}$ \\
\hline \multirow{2}{*}{ Total } & Conversar & $1,73(1,61)$ & $2,32(3,44)$ & - & $0,017^{*}$ \\
& & & & 2,418 & \\
\cline { 2 - 6 } & Ajudar & $0,58(0,67)$ & $0,36(0,60)$ & 2,080 & $0,039^{*}$ \\
\hline \multirow{2}{*}{ Ensino Infantil } & Conversar & $1,87(1,50)$ & $2,70(1,52)$ & - & $0,017^{*}$ \\
& & & & 2,429 & \\
\cline { 2 - 6 } & Agradecer & $0,47(0,75)$ & $0,43(0,71)$ & - & $0,041^{*}$ \\
& & & & 2,077 & \\
\hline
\end{tabular}

Ensino Fundamental

Não houve diferença entre os grupos em nenhuma categoria.

Legenda: *Diferenças significativas no nível de $p \leq 0,05$;** Com problemas de comportamento clínico (CBCL); *** Sem problemas de comportamento clínico (CBCL).

Fonte: Elaborada pelas autoras.

A Tabela 3 refere-se à comparação das habilidades sociais de crianças CPC e SPC. A habilidade conversar apresentou maior média para SPC na amostra total e na do ensino infantil (EI). Já as variáveis ajudar e agradecer obtiveram maiores médias para os CPC na amostra total e na subamostra do ensino infantil (EI). Deve-se pontuar que a diferença das médias da variável agradecer para grupos CPC e SPC foi muito sutil. 
Tabela 4. Habilidades sociais de crianças do ensino infantil (El) e do ensino fundamental (EF), diferenciados por meninos e meninas

\begin{tabular}{cccccc}
\hline Categorias específicas do RE-HSE-P & $\begin{array}{c}\text { Menino } \\
(\mathbf{n}=93)\end{array}$ & $\begin{array}{c}\text { Menina } \\
(\mathbf{n}=62)\end{array}$ & \\
\cline { 3 - 6 } & & \multicolumn{2}{c}{ Média (Desvio Padrão) } & $\boldsymbol{t}$ & $\boldsymbol{p}$ \\
\hline Total & Conversar & $1,77(1,3)$ & $2,40(1,75)$ & $-2,380$ & $0,019^{*}$ \\
\hline \multirow{2}{*}{ Ensino Infantil } & Conversar & $1,95(1,25)$ & $2,69(1,80)$ & $-2,084$ & $0,042^{*}$ \\
\cline { 2 - 6 } & Ser assertivo & $1,90(1,19)$ & $2,58(1,44)$ & $-2,245$ & $0,028^{*}$ \\
\hline Ensino Fundamental & Desculpar-se & $0,08(0,27)$ & $0,34(0,62)$ & 30,03 & $0,048^{*}$ \\
\cline { 2 - 6 } & & & & &
\end{tabular}

Legenda: *Diferenças significativas no nível de $p \leq 0,05$.

Fonte: Elaborada pelas autoras.

Na Tabela 4, é possível observar que todas as habilidades apresentaram maior média entre as meninas. Conversar apareceu para amostra total e para subamostra do El, ser assertivo ocorreu também para o El e desculpa-ser para o EF.

Tabela 5. Queixas de problemas de comportamento de crianças do ensino infantil (EI) e do ensino fundamental (EF), diferenciados pela presença ou ausência de problemas de comportamento

\begin{tabular}{cccccc}
\hline Categorias gerais do RE-HSE-P & \multicolumn{2}{c}{$\begin{array}{c}\text { CPC** } \\
(\mathbf{n}=79)\end{array}$} & $\begin{array}{c}\text { SPC } \\
(\mathbf{n}=76)\end{array}$ & & \\
\cline { 2 - 6 } & & \multicolumn{2}{c}{ Média (Desvio Padrão) } & $\boldsymbol{t}$ & $\boldsymbol{p}$ \\
\hline \multirow{3}{*}{ Total } & Desobedecer & $1,10(1,19)$ & $0,59(0,81)$ & 3,106 & $0,002^{*}$ \\
\cline { 2 - 6 } & Responder mal & $0,79(1,21)$ & $0,39(0,71)$ & 2,531 & $0,013^{*}$ \\
\cline { 2 - 6 } & Demonstrar & $0,91(1,14)$ & $0,55(0,82)$ & 2,246 & $0,026^{*}$ \\
& raiva & & & 2,573 & $0,012^{*}$ \\
\hline \multirow{2}{*}{ Ensino Infantil } & Desobedecer & $1,12(0,95)$ & $0,60(0,87)$ & 2,222 & $0,030^{*}$ \\
\cline { 2 - 6 } & Demonstrar & $1,02(1,13)$ & $0,55(0,71)$ & & \\
\hline
\end{tabular}


Ensino

Não houve diferença entre os grupos em nenhuma categoria.

Fundamental

Legenda: *Diferenças significativas no nível de $p \leq 0,05$; ** Com problemas de comportamento clínico (CBCL); *** Sem problemas de comportamento clínico (CBCL).

Fonte: Elaborada pelas autoras.

$\mathrm{Na}$ tabela 5 , todas as queixas de problemas de comportamento apresentaram maiores médias para as crianças CPC. As variáveis desobedecer, responder mal e demonstrar raiva apareceram na amostra total. Na subamostra do El, encontrou-se apenas desobedecer e demonstrar raiva.

Tabela 6. Queixas de problemas de comportamento de crianças do ensino infantil (EI) e do ensino fundamental (EF), diferenciados por meninos e meninas

\begin{tabular}{|c|c|c|c|c|c|}
\hline \multicolumn{2}{|c|}{ Categorias gerais do RE-HSE-P } & \multicolumn{4}{|c|}{$\begin{array}{l}\text { Menina } \\
(n=62)\end{array}$} \\
\hline & & \multicolumn{2}{|c|}{ Média (Desvio Padrão) } & \multirow{2}{*}{$\begin{array}{c}\boldsymbol{t} \\
2,043\end{array}$} & \multirow{2}{*}{$\begin{array}{c}p \\
0,043\end{array}$} \\
\hline \multirow{5}{*}{ Total } & Agredir & $0,17(0,40)$ & $0,06(0,24)$ & & \\
\hline & Responder mal & $0,75(1,16)$ & $0,37(0,68)$ & 2,563 & 0,011 \\
\hline & Ficar quieto & $0,83(1,05)$ & $0,51(0,95)$ & 1,976 & 0,050 \\
\hline & Esquivar-se & $1,04(1,42)$ & $0,38(0,73)$ & 3,764 & 0,000 \\
\hline & Raiva & $0,88(1,08)$ & $0,51(0,86)$ & 2,330 & 0,021 \\
\hline \multirow{5}{*}{ Ensino Infantil } & Agredir & $0,30(0,51)$ & $0,11(0,31)$ & 2,021 & 0,047 \\
\hline & Fazer birra & $2,37(2,17)$ & $1,44(, 125)$ & 2,370 & 0,021 \\
\hline & Responder mal & $0,79(1,31)$ & $0,30(0,52)$ & 2,211 & 0,031 \\
\hline & Esquivar-se & $1,39(1,67)$ & $0,52(0,77)$ & 3,027 & 0,004 \\
\hline & Raiva & $1,04(1,06)$ & $0,47(0,73)$ & 2,816 & 0,006 \\
\hline \multirow{3}{*}{$\begin{array}{c}\text { Ensino } \\
\text { Fundamental }\end{array}$} & Ficar quieto & $0,94(1,18)$ & $0,46(0,81)$ & 2,070 & 0,042 \\
\hline & Esquivar-se & $0,74(1,08)$ & $0,19(0,63)$ & 2,775 & 0,007 \\
\hline & Ficar tímido & $0,46(0,90)$ & $0,15(0,46)$ & 1,945 & 0,056 \\
\hline
\end{tabular}

Diferenças significativas no nível de $p \leq 0,05$.

Fonte: Elaborada pelas autoras. 
Os dados da Tabela 6 demonstram que as queixas de problemas de comportamento ocorreram para os meninos, independentemente da fase escolar, e o comportamento esquivar-se foi comum aos três grupos. Também se pode notar que houve maior número de queixas com médias maiores para as crianças do El e para amostra total, especialmente referente aos comportamentos agressivos.

Tabela 7. Comparação das práticas negativas das mães de crianças do ensino infantil (El) e do ensino fundamental (EF), diferenciadas pela presença ou ausência de problemas de comportamento clínico $(\mathrm{CBCL})$

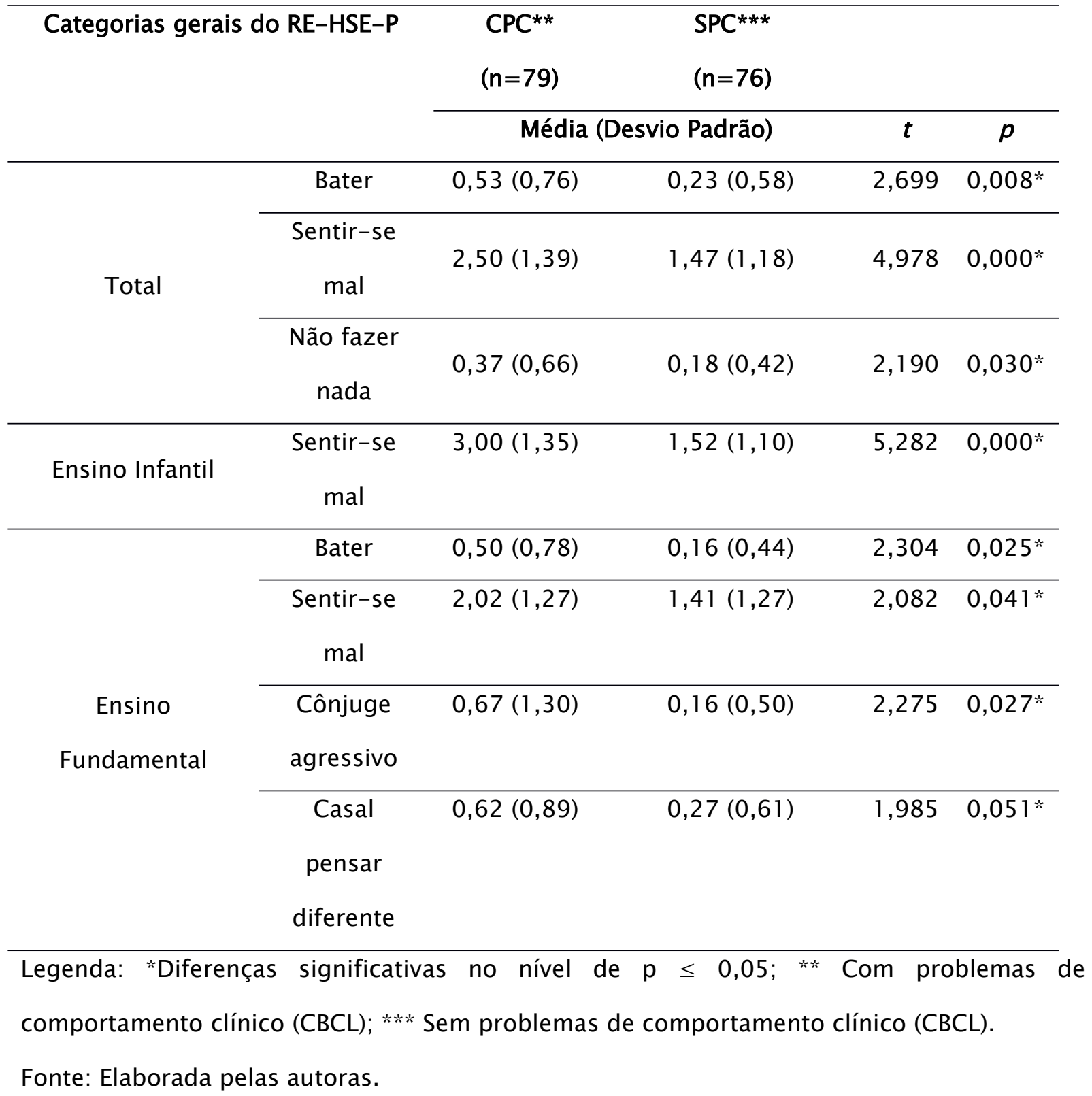


Os dados da Tabela 7 apresentam que as médias das práticas negativas foram maiores para o grupo CPC, independentemente da fase escolar. A prática sentir-se mal apareceu em todos os grupos, seguida de bater (que não apareceu para o El). Para a subamostra do EF, houve maior número de práticas negativas (bater, sentir-se mal, cônjuge agressivo e casal pensar diferente).

Tabela 8. Comparação das práticas negativas das mães de crianças do ensino infantil (EI) e do ensino fundamental (EF), diferenciadas por meninos e meninas

\begin{tabular}{|c|c|c|c|c|c|}
\hline \multicolumn{2}{|c|}{ Categorias gerais do RE-HSE-P } & \multirow{2}{*}{$\begin{array}{c}\text { Menino }(n=93) \\
\text { Média (Desvi }\end{array}$} & $\begin{array}{l}\text { Menina } \\
(n=62)\end{array}$ & \multirow[b]{2}{*}{$t$} & \multirow{3}{*}{$\begin{array}{c}p \\
0,004\end{array}$} \\
\hline & & & o Padrão) & & \\
\hline \multirow{6}{*}{ Total } & Bater & $0,50(0,80)$ & $0,20(0,44)$ & 2,932 & \\
\hline & Colocar de castigo & $0,96(1,14)$ & $0,54(0,80)$ & 2,677 & 0,008 \\
\hline & Sentir-se mal & $2,20(1,37)$ & $1,69(1,37)$ & 2,269 & 0,025 \\
\hline & Cônjuge agressivo & $0,55(0,28)$ & $0,16(0,12)$ & 3,279 & 0,001 \\
\hline & $\begin{array}{l}\text { Expressar-se na } \\
\text { presença do filho }\end{array}$ & $0,08(1,17)$ & $0,12(0,38)$ & 2,093 & 0,038 \\
\hline & Brigar com a criança & $0,64(0,85)$ & $0,95(1,03)$ & 1,938 & 0,055 \\
\hline \multirow{4}{*}{ Ensino Infantil } & Bater & $0,60(0,84)$ & $0,22(0,48)$ & 2,506 & 0,015 \\
\hline & Colocar de castigo & $1,27(1,41)$ & $0,69(0,88)$ & 2,229 & 0,029 \\
\hline & Sentir-se mal & $2,53(1,43)$ & $1,91(1,38)$ & 1,945 & 0,055 \\
\hline & Cônjuge agressivo & $0,53(1,12)$ & $0,11(0,39)$ & 2,312 & 0,025 \\
\hline \multirow{3}{*}{$\begin{array}{l}\text { Ensino } \\
\text { Fundamental }\end{array}$} & Colocar de castigo & $0,70(0,76)$ & $0,34(0,62)$ & 2,160 & 0,035 \\
\hline & Cônjuge agressivo & $0,58(1,23)$ & $0,15(0,36)$ & 2,262 & 0,027 \\
\hline & Brigar com a criança & $0,52(0,78)$ & $1,11(1,07)$ & 2,504 & 0,017 \\
\hline
\end{tabular}

Diferenças significativas no nível de $p \leq 0,05$.

Fonte: Elaborada pelas autoras. 
A Tabela 8 apresenta os resultados referentes às práticas negativas. Nota-se que bater, colocar de castigo, sentir-se mal e cônjuge agressivo obteve maior média entre os meninos; já brigar e expressar-se na frente da criança, entre as meninas da amostra total. Quanto ao El, bater, colocar de castigo, sentir-se mal e cônjuge agressivo foi mais frequente para os meninos. Para a subamostra do EF, colocar de castigo e cônjuge agressivo também apresentou maior média para os meninos; e brigar com a criança para as meninas.

Tabela 9. Comparação das práticas positivas das mães de crianças do ensino infantil (El) e do ensino fundamental (EF), diferenciadas pela presença ou ausência de problemas de comportamento clínico (CBCL)

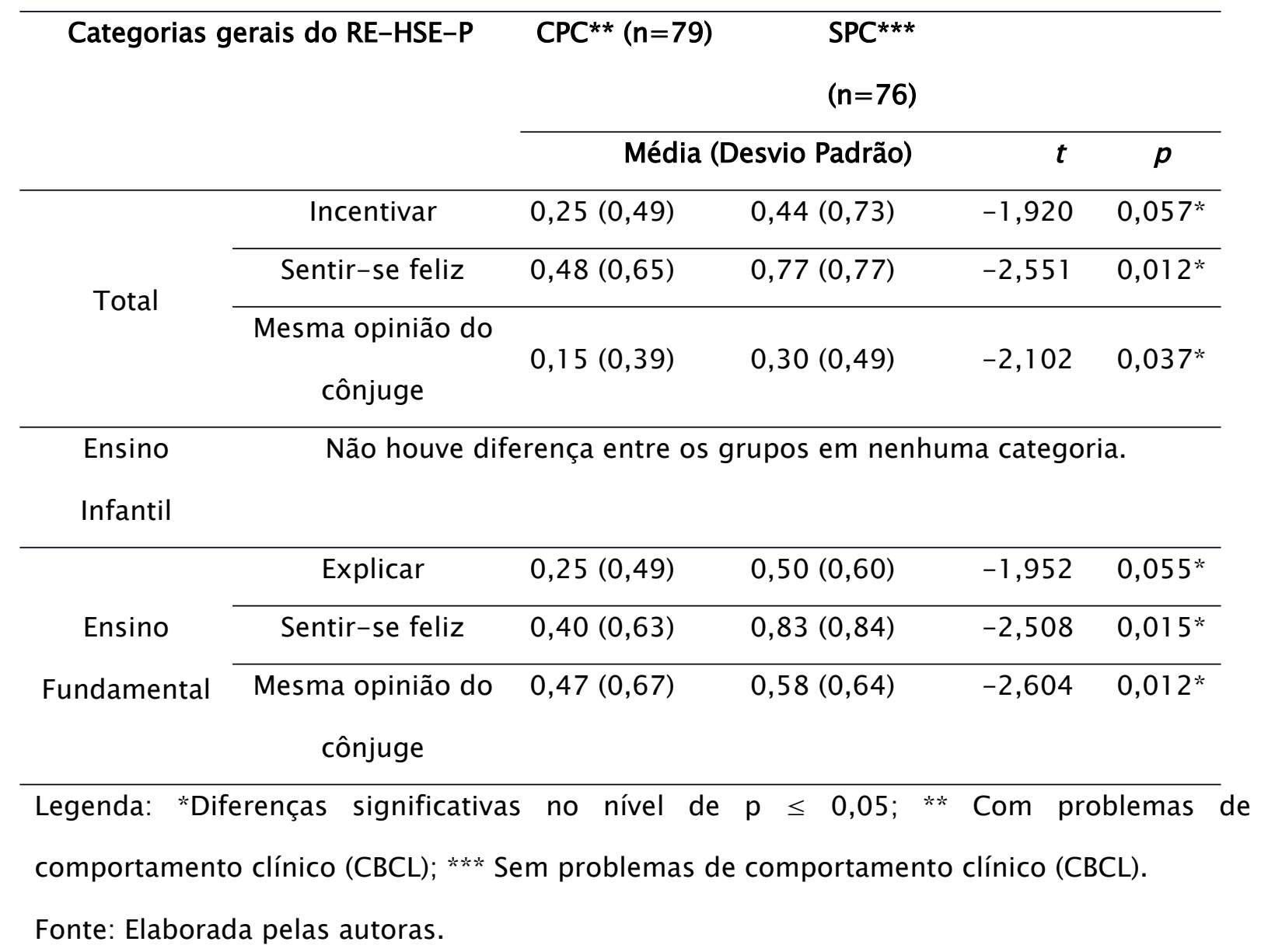

A Tabela 9 demonstra que as médias das práticas positivas foram maiores para o grupo SPC. As práticas positivas sentir-se feliz e ter a mesma opinião que o cônjuge 
apareceu na amostra total e na subamostra do EF. As práticas incentivar e explicar ocorreram para a amostra total e EF, respectivamente.

Tabela 10. Comparação das práticas positivas das mães de crianças do ensino infantil (EI) e do ensino fundamental (EF), diferenciadas por meninos e meninas

\begin{tabular}{|c|c|c|c|c|c|}
\hline Categorias & erais do RE-HSE-P & \multicolumn{2}{|c|}{$\begin{array}{ll}\text { Menino }(n=93) & \text { Menina } \\
& (n=62)\end{array}$} & \multirow[b]{2}{*}{$t$} & \multirow{3}{*}{$\begin{array}{c}p \\
0,010\end{array}$} \\
\hline & & \multicolumn{2}{|c|}{ Média (Desvio Padrão) } & & \\
\hline \multirow{4}{*}{ Total } & Sentir-se feliz & $0,49(0,61)$ & $0,82(0,84)$ & $-2,634$ & \\
\hline & Desculpar-se & $0,29(0,54)$ & $0,51(0,80)$ & $-1,935$ & 0,056 \\
\hline & $\begin{array}{l}\text { Mesma opinião do } \\
\text { cônjuge }\end{array}$ & $0,16(0,39)$ & $0,32(0,50)$ & $-2,115$ & 0,037 \\
\hline & Sentir-se feliz & $0,44(0,61)$ & $0,92(0,93)$ & $-2,383$ & 0,022 \\
\hline Ensino Infantil & $\begin{array}{l}\text { Mesma opinião do } \\
\text { cônjuge }\end{array}$ & $0,16(0,43)$ & $0,38(0,54)$ & $-2,004$ & 0,049 \\
\hline $\begin{array}{l}\text { Ensino } \\
\text { Fundamental }\end{array}$ & \multicolumn{5}{|c|}{ Não houve diferença entre os grupos em nenhuma categoria. } \\
\hline
\end{tabular}

Diferenças significativas ao nível de $p \leq 00,5$.

$\mathrm{Na}$ Tabela 10, é possível observar que as práticas positivas sentir-se feliz, desculpar-se e ter a mesma opinião que o cônjuge diferenciaram grupos, com maiores médias para as meninas. Entre os do El, ter a mesma opinião que o cônjuge também apresentou maior media entre as meninas.

\section{Discussão}

Este estudo buscou comparar os comportamentos presentes na relação mães e filhos do ensino infantil (EI) e fundamental (EF), diferenciadas pela presença ou ausência de problemas de comportamento e, posteriormente, por grupos de meninos e meninas. Os dados encontrados demonstraram que há diferenças nas práticas educativas maternas e nos 
comportamentos emitidos pelas crianças, quando analisados os subgrupos amostrais (escolaridade e sexo; escolaridade e presença/ausência de problemas de comportamento), como observado também em outros estudos (Alvarenga et al., 2016; Bolsoni-Silva \& Loureiro, 2016; Marin et al., 2012).

Inicialmente, os problemas de comportamento não diferenciaram crianças apenas pela fase escolar, o que contraria alguns estudos, como os de Pizato et al. (2014) e de Reynolds et al. (2010), que constataram diferenças. As divergências podem ter aparecido principalmente devido a diferenças metodológicas. Pizato et al. (2014) buscaram avaliar a trajetória de habilidades sociais num estudo longitudinal, o que o presente estudo não se propôs a fazer. Além disso, Pizato et al. (2014) utilizaram instrumento de rastreamento para avaliar problemas de comportamento, diferentemente do presente estudo, que utilizou o $\mathrm{CBCL}$, que avalia a partir de indicadores clínicos.

Por outro lado, na comparação entre meninos e meninas, problema de comportamento clínico apresentou maiores médias para os meninos, tanto do El quanto do EF, corroborando a literatura (Emerich, Rocha, Silvares \& Gonçalves, 2012; Reynolds et al.,2010), que afirma que os meninos têm mais problemas de comportamento que as meninas e são, certamente, expostos a mais fatores de risco, como as práticas negativas (Leman \& Bjornberg, 2010).

Com relação às habilidades sociais infantis, em sua maioria, as médias foram maiores para as meninas e para as crianças SPC, novamente reafirmando outras investigações (Bolsoni-Silva et al., 2016; Emerich et al., 2012; Reyna \& Silvina, 2015), mas contrariando o estudo de Simões e Castro (2018), que não encontraram diferenças nas habilidades sociais de meninos e meninas, o que pode ser explicado devido a diferenças metodológicos e de instrumentos.

A habilidade social infantil mais frequente foi conversar, para subamostra do El e para a amostra total, o que corrobora Bolsoni-Silva e Loureiro (2016), que descobriram mais habilidades sociais relacionadas à comunicação com crianças do El. Pode ser que os filhos menores busquem mais atenção das mães por demandarem mais ajuda e monitoria dos 
seus comportamentos, evidenciando, para esse grupo, as habilidades referentes à conversação.

A respeito das crianças do EF, a única habilidade social que diferenciou os grupos foi desculpar-se e ocorreu mais para as meninas. No estudo de Andersson e Sommerfelt (2001), as mães utilizavam mais práticas positivas com as meninas por esperarem delas comportamentos mais tranquilos e controláveis relacionados à polidez; desse modo, supõem que desculpar-se pode ser mais cobrado das filhas do que dos filhos.

Nessa direção, vão também os dados do presente estudo a respeito das práticas positivas. A prática positiva desculpar-se foi mais frequente para as meninas do EF, e explicar com as crianças SPC, também do EF, diferenciando os grupos. Esses resultados indicam que as mães das meninas maiores provavelmente se comunicam mais com elas, mesmo diante dos comportamentos indesejados, inclusive para dar explicações. Além disso, é bem provável que elas ofereçam modelos de como se desculpar, o que pode favorecer a emissão desse mesmo comportamento pelas filhas.

Outra habilidade social com maior média para as meninas foi ser assertivo, para as maiores, e oferecer ajuda, para a amostra como um todo, corroborando Simão e Castro (2018), que também encontraram níveis mais altos de assertividade para meninas do fundamental, associado a menores níveis de psicotismo. Nessa direção, os resultados do presente estudo também demonstraram que as mães das meninas do EF e da amostra total costumam sentir felicidade na interação com elas e concordar mais com o cônjuge, elas também incentivam mais as filhas, se comparadas às interações com os meninos. As relações encontradas corroboram estudos (Bolsoni-Silva et al., 2016; Emerich et al., 2012; Reyna \& Silvina, 2015) que afirmam que as práticas positivas são preditores de habilidades sociais infantis e que costumam aparecer mais na interação com as meninas.

A assertividade diz respeito às habilidades de enfrentamento (Del Prette $\&$ Del Prette, 2001), cujo desenvolvimento pode ser encorajado por meio de comunicação assertiva e modelo (Del Prette \& Del Prette, 2017); além disso, a maior concordância entre os genitores quanto à educação das filhas demonstra consistência parental, o que favorece o estabelecimento de limites e a compreensão pela criança dos comportamentos que são 
esperados (Bolsoni-Silva \& Marturano, 2010), reduzindo a probabilidade de problemas de comportamento (Marin, Martins, Freitas, Lopes \& Piccinini, 2013).

A respeito da categoria queixas de problemas de comportamento, as variáveis apresentaram médias mais elevadas para os CPC e meninos, novamente concordando com a literatura (Bolsoni-Silva et al., 2016; Teixeira et al., 2015; Torre et al., 2013). Para as crianças CPC e meninos do El, os problemas mais relatados (desobedecer, demonstrar raiva, responder mal, agredir, fazer birra) podem ser identificados como externalizantes. É possível que, por se tratarem de crianças pequenas, cujo autocontrole ainda é pouco desenvolvido (Konold et al., 2010; Papalia, 2006; Pizato et al., 2014), mais problemas de comportamento antissociais, irritáveis e impulsivos sejam encontrados (Bolsoni-Silva, 2003).

O contrário ocorreu para os meninos do EF, para quem a maior parte dos comportamentos identificados foi internalizantes (ficar quieto e ser tímido) - com exceção de esquivar-se -, que pode ser enquadrado nos externalizante e/ou internalizantes, a depender da sua topografia. Casali-Robalinho, Del Prette e Del Prette (2015) também encontraram mais problemas internalizantes para crianças do ensino fundamental, cujos pais indicaram dificuldades dos filhos relacionadas à comunicação e iniciativa social, deficit que podem ser relacionados à timidez e quietude. Uma explicação para isso é o fato de as crianças mais velhas estarem muito envolvidas com a tecnologia e disporem de pouco investimento nas relações sociais, o que pode maximizar deficit habilidosos e diminuir oportunidade de aprender comportamentos socialmente relevantes (Casali-Robalinho et al., 2015; Espinar, Ruiz \& López Fernández, 2009).

Ademais, esquivar-se foi encontrado para meninos do El e do EF, indicando recorrência para ambos os grupos. Comportamentos relacionados à evitação (ou esquiva) podem indicar ansiedade diante da situação social de enfrentamento (Casali-Robalinho et al., 2015), como diante da bronca feita pelos pais ou na resolução de conflitos com os pares.

A respeito das práticas negativas, sentir-mal diferenciou grupos, com média mais elevada para crianças com problemas do El, e as práticas bater e não fazer nada apareceram 
para a amostra total com problemas. Como já foi dito, as crianças mais novas apresentam demandas diferentes das mais velhas, especialmente relacionadas a habilidades de socialização (Papalia, 2006), assim, pode ser que faltem HSE-P às mães para conversar e estabelecer regras que os filhos consigam compreender, gerando uma condição de malestar ou impotência diante das demandas das crianças.

Nas análises das práticas negativas direcionadas às crianças do EF com problemas, as mães relataram comportamentos como bater, cônjuge ser agressivo, casal pensar diferente e colocar de castigo, sugerindo que elas utilizam mais práticas agressivas com as crianças CPC, especialmente se forem mais velhas, revelando ainda que mães e pais podem discordar sobre a melhor forma de lidar com as demandas do filhos, fator que não favorece o desempenho habilidoso da criança, sobretudo no ambiente familiar (Bolsoni-Silva \& Marturano, 2010; Marin et al., 2013).

Quanto ao sexo, para os meninos, práticas negativas como colocar de castigo e agressividade do cônjuge foram frequentes, para a amostra do ensino infantil e fundamental, mas práticas como bater, sentir-mal obtiveram maior média apenas para o El. Ou seja, as mães de meninos emitem comportamentos agressivos com mais frequência na comparação com as das meninas, assim como apareceu no estudo de Assis (2017). Deve-se pontuar a tendência dos meninos de demonstrarem mais problemas comportamentais do que as meninas, exigindo mais HSE-P e manejo comportamental para lidar com tais demandas (Cosentino-Rocha \& Linhares, 2013), repertório que pode faltar às mães do presente estudo.

\section{Considerações finais}

A presente pesquisa estudou a relação entre comportamentos maternos e infantis, diferenciados por subgrupos definidos a partir da fase escolar, sexo da criança e presença/ausência de problema de comportamento. Os achados deste estudo contribuíram para responder a questões ainda em aberto na literatura relacionadas às diferenças 
comportamentais de crianças do El e EF e às práticas maternas direcionadas às crianças de diferentes idades, sexo e com e sem problemas clínicos.

Em suma, a pesquisa não encontrou relação entre os problemas de comportamento clínico e etapa escolar da criança para a amostra total, mas constatou que os meninos, fossem do ensino infantil, fossem do fundamental, tinham mais problemas de comportamento. Também se verificou que as práticas positivas e as habilidades sociais infantis apareceram para o grupo de meninas e para as crianças sem problemas de comportamento, e as práticas negativas e queixas de problemas de comportamento para os meninos e crianças com problemas de comportamento, independentemente da fase escolar.

Enquanto contribuições para a área, o estudo encontrou que: a) o comportamento de conversar foi a habilidade social que mais apareceu e foi mais frequente para as crianças do El, porém, pedir desculpas ocorreu mais para as meninas do EF; b) há maior variedade de práticas negativas que apareceram nas análises que inclui o grupo de meninos do que o grupo com problemas de comportamento; c) entre as práticas negativas, as que mais apareceram foram o cônjuge ser agressivo, sentir-se mal e colocar de castigo; d) sobre as queixas de problemas de comportamento, houve mais queixas relacionadas aos problemas externalizantes para os meninos do El e mais internalizantes para os meninos do EF; e) quanto às práticas positivas, as que mais apareceram foram concordar com o cônjuge e sentir-se feliz.

Vale destacar que a complexidade presente nas interações mães-filhos demanda estudos que busquem compreender e caracterizar funcionalmente os comportamentos presentes, a fim de subsidiar programas de intervenções mais eficazes e efetivos.

Enquanto limitações, a pesquisa contou com o relato apenas das mães das crianças, o que pode limitar a análise dos contextos de interações; além disso, o estudo utilizou instrumentos apenas de relato verbal, sem uso de observação direta para confirmação e melhor compreensão dos relatos obtidos. E também as amostras do ensino infantil e fundamental, meninos e meninas, com e sem problemas de comportamento não foram equilibradas, o que pode ser foco de estudo futuro. 


\section{Fonte de financiamento}

Estudo financiado pela Fundação de Amparo à Pesquisa do Estado de São Paulo (FAPESP), por meio do processo $n^{\circ} 2016 / 01264-0$.

\section{Referências}

Achenbach, T. M., \& Rescorla, L. A. (2001). Manual for the Achenbach System of Empirically Based Assessment School-Age Forms Profiles. Burlington, VT: Aseba.

Alvarenga, P. A., Weber, L. N. D., \&Bolsoni-Silva, A. T. (2016). Cuidados parentais e desenvolvimento socioemocional na infância e na adolescência: uma perspectiva analítico-comportamental. Revista Brasileira de Terapia Comportamental e Cognitiva, 18(1). https://doi.org/10.31505/rbtcc.v18i1.827.

Andersson, H. W., \& Sommerfelt, K. (2001).The Relationship between Cognitive Abilities and Maternal Ratings of Externalizing Behaviors in Preschool Children. Scandinavian Journal of Psychology, 42(5), 437-444. https://doi.org/10.1111/1467-9450.00256.

Assis, R. P. (2017). Práticas educativas, problemas de comportamento e habilidades sociais infantis: um estudo comparativo e correlacional de medidas de relato. Dissertação de mestrado, Universidade Estadual Paulista Julio de Mesquista Filho, Bauru, São Paulo, Brasil.

Bartholomeu, D., Montiel, J. M., Fiamenghi Jr., G. A., \& Machado, A. A. (2016, Apr./jun.). Predictive Power of Parenting Styles on Children's Social Skills: a Brazilian Sample. SAGE Open, 1-7. http:// dx.doi.org: 10.1177/2158244016638393.

Bolsoni-Silva, A. T. (2003). Habilidades sociais educativas, variáveis contextuais e problemas de comportamento: comparando pais e mães de escolares. Tese de doutorado, Departamento de Psicologia, Universidade de São Paulo, Ribeirão Preto, São Paulo. 
Bolsoni-Silva, A. T. (2017). Práticas parentais educativas na interação social mães-filhos. Revista Brasileira de Terapia Comportamental e Cognitiva, 19(4), 24-44. https://doi.org/10.31505/rbtcc.v19i4.1092.

Bolsoni-Silva, A. T., \& Loureiro, S. R. (2016). Simultaneous Assessment of Social Skills and Behavior Problems: Education and Gender. Estudos de Psicologia (Campinas), 33(3), 453-464. http://dx.doi.org/10.1590/1982-02752016000300009.

Bolsoni-Silva, A. T., Loureiro, S. R., \& Marturano, E. M. (2016). Roteiro de Entrevista de Habilidades Sociais Educativas Parentais (Re-Hse-P). São Paulo: Hogrefe Cetepp. (Teste Psicológico).

Bolsoni-Silva, A. T., \& Marturano, E. M. (2010). Relacionamento conjugal, problemas de comportamento e habilidades sociais de pré-escolares. Psicologia: teoria e pesquisa, $26(1), 67$.

Bolsoni-Silva, A. T., Marturano, E. M., \& Freiria, L. R. B. (2010). Indicativos de problemas de comportamento e de habilidades sociais em crianças: um estudo longitudinal. Psicologia: Reflexão e Crítica, 23(3), 506-515. http://dx.doi.org//10.1590/S010279722010000300011 .

Bolsoni-Silva, A. T., Marturano, E. M., \& Loureiro, S. R. (2011). Estudos de confiabilidade e validade do Questionário de Respostas Socialmente Habilidosas: Versão para Pais QRSH-PAIS-Pais. Psicologia: Reflexão e Crítica, 24(2), 1-9. http://dx.doi.org//10.1590/S0102-79722011000200003.

Casali-Robalinho, I. G., Del Prette, Z. A. P., \& Del Prette, A. (2015). Habilidades sociais como preditoras para problemas de comportamento em escolares. Psicologia: Teoria e Pesquisa, $\quad 31(3), \quad 321-330 . h t$ ttp://dx.doi.org/10.1590/010237722015032110321330. 
Comôdo, C. N., Del Prette, A., \& Del Prette, Z. A. P. (2017). Intergeracionalidade das habilidades sociais entre pais e filhos adolescentes. Psicologia: Teoria e Pesquisa, 33(1). http://dx.doi.org/10.1590/0102.3772e33311.

Cosentino-Rocha, L., \& Linhares, M. B. M. (2013). Temperamento de crianças e diferença de gênero. Paidéia, 23(54), 63-72. http://dx.doi.org/10.1590/1982-43272354201308.

Del Prette, A., \& Del Prette, Z. A. P. (2001). Psicologia das relações interpessoais e habilidades sociais: vivências para o trabalho em grupo. Petrópolis: Vozes.

Del Prette, A., \& Del Prette, Z. A. P. (2017). Competência social e habilidades sociais: manual teórico-prático. Petrópolis: Editora Vozes.

Dutra, A. (2010). Esquiva experiencial na relação terapêutica. In A. K. C. R. Farias. Análise comportamental clinica: aspectos teóricos e estudos de caso (pp. 201-214). Porto Alegre: Artmed.

Emerich, D. R., Rocha, M. M., Silvares, E. F. M., \& Gonçalves, J. P. (2012, jul./set.). Diferenças quanto ao gênero entre escolares brasileiros avaliados pelo Inventário de Comportamentos para Crianças e Adolescentes (CBCL/6-18). Psico, 43(3), 380-387.

Espinar Ruiz, E., \& López Fernández, C. (2009). Jóvenes y adolescentes ante las nuevas tecnologías: percepción de riesgos. Athenea Digital, 16, 1-20. http://doi.org// 10.5565/rev/athenead/v0n16.509.

Gomide, P. I. C. (2006). Manual do inventário de estilos parentais: modelo teórico, manual de aplicação, apuração e interpretação. Petrópolis, RJ: Vozes.

Kårstad, S. B., Kvello, Ø., Wichstrøm, L., \& Berg-Nielsen, T. S. (2014). What do Parents Know about their Children's Comprehension of Emotions?: Accuracy of Parental Estimates in a Community Sample of Pre-Schoolers. Child: care, health and development, 40(3), 346-353. https://doi.org/10.1111/cch.12071. 
Kim, M., Doh, H., Hong, J. H., \& Choi, M. (2011). Social Skills Training and Parent Education Programs for Aggressive Preschoolers and their Parents in South Korea. Children and $\begin{array}{llll}\text { Youth Service } & \text { Review, } & \text { 838-845), }\end{array}$ https://doi.org/10.1016/j.childyouth.2010.12.001.

Konold, T. R., Jamison, K. R., Stanton-Chapman, T. L., \& Rimm-Kaufman, S. E. (2010). Relationships among Informant Based Measures of Social Skills and Student Achievement: a Longitudinal Examination of Differential Effects by Sex. Applied Developmental Science, $14(1)$, $8-34$. https://doi.org/10.1080/10888690903510307.

Leman, P. J., \& Björnberg, M. (2010). Conversation, Development, and Gender: a Study of Changes in Children's Concepts of Punishment. Child Development, 81(3), 958-971. https://doi.org/10.1111/j.1467-8624.2010.01445.x.

Leme, V. B. R., \& Marturano, E. M. (2014). Preditores de comportamentos e competência acadêmica de crianças de famílias nucleares, monoparentais e recasadas. Psicologia: Reflexão e Crítica, 27(1).

Marin, A. H., Martins, G. D. F., Freitas, A. P. C. O., Silva, I. M., Lopes, R. D. C. S., \& Piccinini, C. A. (2013). Transmissão intergeracional de práticas educativas parentais: evidências empíricas. Psicologia: Teoria e Pesquisa, 29(2), 123-132.

Marin, A. H., Piccinini, C. A., Gonçalves, T. R., \&Tudge, J. R. (2012). Práticas educativas parentais, problemas de comportamento e competência social de crianças em idade pré-escolar. Estudos de Psicologia, 17(1).

Newcombe, N. (1999). Desenvolvimento infantil: abordagem de Mussen. Porto Alegre: Artmed. 
Patias, N. D., Siqueira, A. C., \& Dias, A. C. G. (2013). Práticas educativas e intervenção com pais: a Educação como proteção ao desenvolvimento dos filhos. Mudanças Psicologia e Saúde, 21(1), 29-40.

Papalia, D., Olds, S., \& Feldman, R. ( 2006). Desenvolvimento humano. Porto Alegre: Artmed.

Pizato, E. C. G., Marturano, E. M., \& Fontaine, A. M. G. V. (2014). Trajetórias de habilidades sociais e problemas de comportamento no ensino fundamental: influência da Educação infantil. Psicologia: Reflexão e Crítica, 27(1), 189-197.

Reyna, C., \& Brussino, S. (2015). Diferencias de edad y género en comportamiento social, temperamento y regulación emocional en niños argentinos. Acta Colombiana de Psicología, 18(2), 51-64. http://dx.doi.org/10.14718/ACP.2015.18.2.5.

Reynolds, M. R., Sander, J. B., \& Irvin, M. J. (2010). Latent Curve Modeling of Internalizing Behaviors and Interpersonal Skills through Elementary School. School Psychology Quarterly, 25,189-201. http://dx.doi.org/10.1037/a0021543.

Sierra, A. V., \& Vega, M. G. P. (2014). El papel de los esquemas cognitivos y estilos de parentales en la relación entre prácticas de crianza y problemas de comportamiento infantil. Avances en Psicología Latinoamericana, 32(3).

Simões, N. C., \& Castro, P. F. (2018). Avaliação psicológica em escolares: relação entre personalidade, autoconceito e habilidades sociais. Gerais: Revista Interinstitucional de Psicologia, $17(1), 26-44$.

Teixeira, M. C. T. V., Marino, R. L. F, \& Carreiro, L. R. R. (2015). Associations between Inadequate Parenting Practices and Behavioral Problems in Children and Adolescents with Attention Deficit Hyperactivity Disorder. The Scientific World Journal. http://dx.doi.org/10.1155/2015/683062.

Torre, M. J., Casanova, P. F., Villa Carpio, M., \& Cerezo, M. (2013). Consistencia e inconsistencia parental: relaciones con la conducta agresiva and satisfacción vital de 
los adolescentes. European Journal of Education and Psychology, 6, 135-149. https://doi.org/10.30552/ejep.v6i2.91.

Recebido em: 9/5/2018

Aprovado em: 6/2/2019 